\title{
HIGHLIGHTS
}

(O) NONINSULIN THERAPIES FOR T1DM

\section{GLP-1 directs differentiation of human embryonic stem cells}

Glucagon-like peptide 1 (GLP-1) could be a key factor driving the development of insulin-producing cells, according to in vitro data published in the journal Pancreas.

The use of islet-cell transplantation to restore pancreatic function in patients with diabetes mellitus is limited by the availability of appropriate donor tissue. An alternative option is to use other cell types, such as human embryonic stem cells, which can be programmed to differentiate into insulin-producing cells. Understanding the signal transduction pathways involved in the differentiation process is vital to the success of this approach.

GLP-1 - a hormone secreted by the L cells of the small intestine-increases pancreatic insulin secretion in response to glucose. In addition, GLP-1 can induce expression of pancreas/duodenum homeobox protein 1 (PDX-1), a transcription factor required for embryonic development of the pancreas. Hongxiang Hui and colleagues, therefore, postulated that treatment of human embryonic stem cells with GLP-1 might induce them to differentiate into cells with the capacity to produce insulin.

The researchers treated the human embryonic stem-cell line HUES1 with exogenous GLP-1 for a period of 21 days. GLP-1 induced differentiation of the HUES1 cells, which was associated with a marked time-dependent downregulation of factors characteristic of stem cells, such as telomerase reverse transcriptase. Conversely, treatment with GLP-1 led to increased levels of PDX-1 and several factors involved in glucose metabolism, including glucokinase, a glucose transporter and insulin. C-peptide and insulin were detected in the culture medium, demonstrating the ability of the differentiated cells to secrete these proteins.

Treatment with GLP-1 also induced expression of components of the hedgehog signal transduction pathway, which is essential for terminal differentiation of the pancreas. Inhibition of the hedgehog pathway with cytopamine markedly reduced the ability of GLP-1 to induce differentiation of the HUES1 cells.

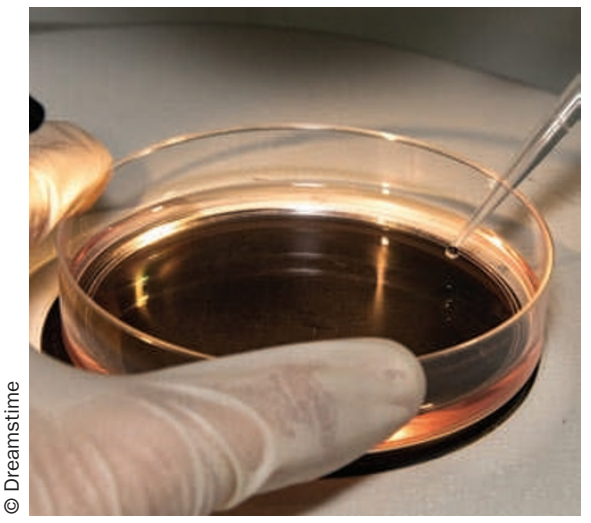

In addition, the differentiationinducing activity of GLP-1 was found to require both the cyclic AMP and phosphatidylinositol-3-kinase signal transduction pathways, but not the mitogen-activated protein kinase pathway.

Hui et al. conclude that their findings shed new light on the process of pancreatic development and may ultimately aid the promotion of stem-cell therapies for patients with diabetes mellitus.

Vicky Heath

Original article Hui, H. et al. Glucagon like peptide-1directed human embryonic stem cells differentiation into insulin-producing cells via Hedgehog, cAMP, and PI3K pathways. Pancreas doi:10.1097/ MPA.0b013e3181bc30dd 